Review

\title{
Phase-transition Perfluorocarbon Nanoparticles for Ultrasound Molecular Imaging and Therapy
}

\author{
Jinshun $\mathrm{Xu}^{1 \# \cong}$, Yang $\mathrm{Cao}^{1,2 \#}$, Chunyan $\mathrm{Xu}^{1}, \mathrm{Xuan}_{\mathrm{Zhou}}^{3}$, Jianxin Liu ${ }^{1}$, Yuanzhi Yao ${ }^{1}, \mathrm{Pan}_{\mathrm{Li}}{ }^{1,2}$, \\ Li Zhou ${ }^{1}$, Yufeng You ${ }^{1}$, Lan Hao ${ }^{1,2}$, Yang Sun ${ }^{1,2}$, Weixiang Song ${ }^{1}$, Yajing Zhao ${ }^{1}$, Zhigang Wang ${ }^{1,2}$ \\ ${ }^{1}$ Institute of Ultrasound Imaging of Chongqing Medical University, Chongqing 400016, China \\ ${ }^{2}$ The Second Affiliated Hospital of Chongqing Medical University, Chongqing 400010, China \\ ${ }^{3}$ Department of Emergency of Chinese PLA General Hospital, China \\ "Both authors contributed equally to this study.
}

\Corresponding author: E-mail: jsxu86@163.com, cqlipan@163.com,wzg62942443@163.com

Received: Feb. 10, 2015; Accepted: Mar. 15, 2015; Published: Mar. 24, 2015.

Citation: Jinshun Xu, Yang Cao, Chunyan Xu, Xuan Zhou, Jianxin Liu, Yuanzhi Yao, Pan Li, Li Zhou, Yufeng You, Lan Hao, Yang Sun, Weixiang Song, Yajing Zhao and Zhigang Wang. Phase-transition Perfluorocarbon Nanoparticles for Ultrasound Molecular Imaging and Therapy. Nano Biomed. Eng. 2015, $7(1), 8-19$.

DOI: $10.5101 /$ nbe.v7il.p8-19.

\begin{abstract}
Recently, the advances in perfluorocarbon nanoparticles have been introduced to expand the diagnostic and therapeutic capability of ultrasound molecular imaging, a non-invasive diagnostic imaging, which passed from robust anatomical presentation to detection of physiological processes and recognition of specific tissue epitopes at the cellular level. The good properties of perfluorocarbon nanoparticles, such as nontoxicity, small size, phase-shift capability, etc., have been resulted in tremendous potential prospects in clinical application. Herein, this review focuses on the mechanisms of perfluorocarbon nanoparticles in terms of phase transition and ultrasonic theranostic. And the potential applications of perfluorocarbon nanoparticles in ultrasound medicine are also discussed.
\end{abstract}

Keywords: Perfluorocarbon Nanoparticle; Molecular Imaging; Ultrasound; Targeted Therapeutics

\section{Introduction}

Ultrasound as a common modality for diagnosis and therapy in clinical medicine due to its intrinsic tissue penetration and high safety [1-3] demonstrates cost-effective, portable, non-ionizing, non-invasive and real-time characteristics compared with other imaging modalities such as X-ray imaging, computed tomography $(\mathrm{CT})$, magnetic resonance imaging (MRI), and positron emission tomography (PET) $[4,5]$. However, the poor quality on imaging and diagnosing of ultrasonography seriously retards further development of its abilities on the detection of diseases in the early stages, particularly for diagnosing tumors. Fortunately, the microbubbles as a contrast agent (CA) have dramatically enhanced diagnostic capabilities of ultrasonography due to their high acoustic impedance for considerable improvement in echogenic properties [6-9]. But the relatively large size of microbubbles (typically two to ten micrometers) does not permit successful extravasation into tumor tissues as a result of the fact that the largest pores existing in the microcirculations of tumors are no more than 700 nanometers [10]. Additionally, the relatively 
large size induces microbubbles to be rapidly captured by the reticuloendothelial systems (RES), leading to the very short blood circulation time (minutes) and low amassing quantities in the tumors [11, 12]. Comparatively, the specially nano-sized bubbles which have overcame this disadvantage in terms of the relatively large size, nevertheless, have just limitedly contributed to enhance the ultrasound imaging because that the nonlinear backscattering is significantly dropped with their diameters decreased [13, 14], and extraordinary image processing algorithms will be needed to increase the signal-to-noise ratio for detection of tumors tissues [15].

During the last decade, advances in nanomedicine have permitted nanoparticles encapsulated various imaging agents, targeting moieties and therapeutic drugs to be a container of various functionalities $[5,16-23]$. One of the most promising examples is the perfluorocarbon (PFC) nanoparticles, because its characters of nontoxic biocompatibility and low solubility in aqueous liquids are extremely essential for medical application [24-27]. The most important thing is that PFC nanoparticles advance a way to solve the above problem for the reason that they would effectively accumulate in tumor tissues beyond to blood vessels and then convert into microbubbles under ultrasound irradiation to enhance the ultrasound imaging [19, 28, 29]. In that case, the ability of PFC nanoparticles to combine imaging efficacy and therapeutic capability may remarkably improve the early assessment of response to treatment and considerably promote the development of personalized therapy $[21,30,31]$. Therefore, in what follows we will focus on PFC nanoparticles in response to the ultrasound-induced phase transition.

\section{The Machanism of Vaporization in PFC Nanoparticles}

Recently, Acoustic droplet vaporization (ADV) has been a considerably exploited phenomenon that liquid droplets are induced to convert into vapors under the action of ultrasound irradiation. It has been known for more than 15 years beginning with a literature by Apfel, who described that a technically designed PFC nanoparticles can transform into microbubbles in the condition of the application of acoustic waves [32]. The name "acoustic droplet vaporization" was given in 2000 by Kripfgans [8]. From then award, ADV has been profoundly investigated in the group works of Michigan University with albumin coated perfluoropentane (PFP) nanoparticles, which illustrated substantially applications PFC nanoparticles with $\mathrm{ADV}$ in terms of drug delivery, thrombolysis, and gas embolism, etc. [33-43].

Table 1 lists some parameters of candidate perfluorocarbons.

\section{Thermodynamics of Vaporization}

The first related concept is the thermodynamic phase state, such as solid, liquid, and gas. Both solid and liquid have a vapor pressure, which increases with the growth of the temperature of the condensed phases. The condensed phase maintains its intrinsic state, liquid or solid, when the vapor pressure is no more than its surrounding pressure. While, once the increase of vapor pressure and/or the decrease of surrounding pressure result in that vapor pressure surpass its surrounding pressure, the molecules will promptly escape from a liquid or solid phase to a gas phase. The "normal boiling point" (as shown in Table 1) is the temperature where the vapor pressure of a PFC liquid is around $101 \mathrm{kPa}$. And during this circumstance, the equilibrium between gas and liquid phases is obtained.

Table 1 Properties of perfluorocarbons

\begin{tabular}{cccc}
\hline Abbreviation & $\begin{array}{c}\text { Chemical } \\
\text { formula }\end{array}$ & Common name & $\begin{array}{c}\text { Normal } \\
\text { boiling point } \\
\left({ }^{\circ} \mathrm{C}\right)\end{array}$ \\
\hline OFP & $\mathrm{C}_{3} \mathrm{~F}_{8}$ & Octafluoropropane & -37 \\
PFB & $\mathrm{C}_{4} \mathrm{~F}_{10}$ & Perfluorobutane & -2 \\
PFP & $\mathrm{C}_{5} \mathrm{~F}_{12}$ & Perfluoropentane & 29 \\
PFH & $\mathrm{C}_{6} \mathrm{~F}_{14}$ & $\begin{array}{c}\text { Perfluorohexane } \\
\text { Perfluorohexyl } \\
\text { bromide }\end{array}$ & 56 \\
PFHB & $\mathrm{C}_{6} \mathrm{~F}_{13} \mathrm{Br}$ & $\begin{array}{c}\text { Perfluorooctyl } \\
\text { bromide }\end{array}$ & 142 \\
PFOB & $\mathrm{C}_{8} \mathrm{~F}_{17} \mathrm{Br}$ & $\begin{array}{c}\text { Perfluoro-15- } \\
\text { crown-5-ether }\end{array}$ & 146 \\
\hline
\end{tabular}

Acoustic droplet vaporization introduces these properties of the thermodynamic phase state to achieve the emergence of phase transformation, not necessarily any changes in temperature, due to the fact that acoustic waves can be used to manipulate the vapor pressure of the liquid. For small droplets trapped by elastic lipid shells, however, even though the vapor pressure of liquid droplets is greater than its surrounding pressure in the surrounding liquid, the small droplets still have not changed to gas because of Laplace pressure, which contributes to the local 
pressure imposed on the interior fluid of droplets due to the surface tension [44-46].

The Laplace pressure is given by

$\Delta P=P_{\text {inside }}-P_{\text {outside }}=2 \gamma / \mathrm{R}$

where $\gamma$ the surface tension, $R$ is droplet radius, Pinside is the pressure inside a droplet, Poutside is the pressure outside a droplet.

Because the surface tension on the interface between water and PFP is estimated to be $56 \pm 1 \mathrm{mN} / \mathrm{m}$ at $37{ }^{\circ} \mathrm{C}$ [47], the Laplace pressure can be calculated to be 224 $\mathrm{kPa}$ when PFP droplets with a diameter of 1 micron is in water with 1 atmosphere pressure and the same temperature. As a result, the internal pressure of droplets is $325 \mathrm{kPa}$, produced by the value of Laplace pressure plus the surrounding water pressure of 101 $\mathrm{kPa}$. Thereby, although the PFP vapor pressure is 132 $\mathrm{kPa}$ at $37^{\circ} \mathrm{C}$, the PFP droplets will never convert into gas because the local internal pressure of droplets remains higher than its vapor pressure. Good examples of increased boiling temperatures of PFC are given by Sheeran et al. [48, 49].

\section{Acoustic Droplet Vaporization}

As a matter of fact, there are no considerable pressure gradients acrossing through the PFC nanoparticle droplets because the wavelengths of ultrasound (propagated in water in $37^{\circ} \mathrm{C}$ ) at $5 \mathrm{MHz}$, $1 \mathrm{MHz}$ are $305 \mu \mathrm{m}, 1.5 \mathrm{~mm}$, respectively, all of which are much larger than the size of droplets. Therefore, even though the internal pressure of droplets is at a higher value due to the Laplace pressure, it will homogeneously rise and fall with the fluctuant pressure of the surrounding fluid, as Fig. 1 shows.

According to the Fig. 1, there are circulated values of internal pressure in the PFC nanoparticle droplets, which increases above the value of vapor pressure of the PFC and then decrease below the value of vapor pressure during a pattern of acoustic pressure cycle. Herein, a time window has been shown when the internal pressure is lower than the vapor pressure. As a result, the gas phase can rapidly formed by the "driving force" if the time window is long enough (low ultrasonic frequency) or the internal pressure is small enough (high acoustic amplitude). These process has been observed by several reports [50-52].

The phase shift capability of PFC nanoparticles will increase with increasing droplet size, ultrasound amplitude, vapor pressure and temperature. The phase

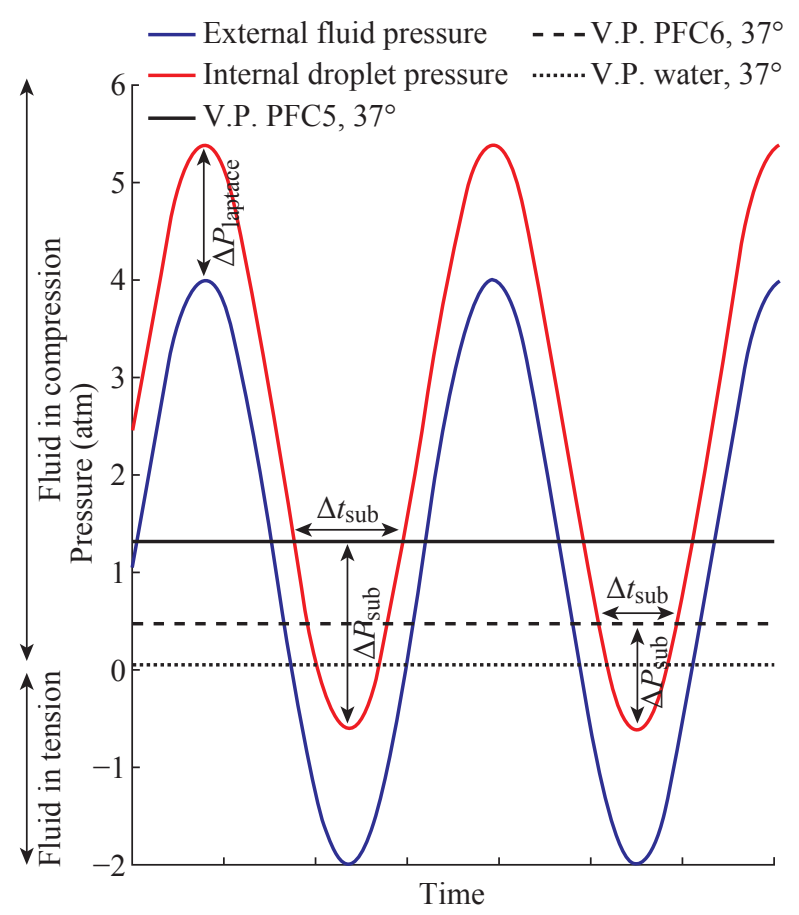

Fig. 1 Upper sinusoidal line represents the internal pressure of a PFC droplet with $1 \mu \mathrm{m}$ in diameter. Lower sinusoidal line represents the surrounding pressure of the fluid under the ultrasound irradiation. The deviation is the Laplace pressure. The vapor pressures of PFP, PFH, and water are indicated at 1 atmosphere pressure. The vertical arrows indicate the maximum difference value between internal pressure and vapor pressure of PFC droplets, and the horizontal arrows indicate the available time window. The plot was adopted with permission from [46].

shift capability of PFC nanoparticles will decrease with increasing ultrasound frequency and surface tension. Many of these results have been confirmed with experimental observations [34, 45, 53].

\section{Bubble Growth}

The gas bubble nucleated will be continuous to grow without the limitation in terms of the heat after the increasing internal pressure of droplets ultimately exceeds the vapor pressure in the ultrasonic field, because the heat is adequate to converted into the energy demanded for satisfying the formation of the gas phase [46]. This circulation of the dynamic system has been reported by Sheeran et al. [45] and observed with PFP and PFH nanoparticle droplets in water at $25{ }^{\circ} \mathrm{C}$ and $37^{\circ} \mathrm{C}$ [46].

The eventual state of the droplets may be a dynamic equilibrium constituted by the expanded gases and condensed liquid droplets after cessation of ultrasound irradiation because the gas phases may persist in some cases. For instance, when the size of PFP droplet 
encapsulated a phosphatidylcholine layer, which is predicted to be a surface tension of $3.5 \mathrm{mN} / \mathrm{m}$ [54], increases from initial $100 \mathrm{~nm}$ to around $517 \mathrm{~nm}$ by $\mathrm{ADV}$, the Laplace pressure is calculated to reduce from $140 \mathrm{kPa}$ to $27 \mathrm{kPa}$ according to the equation (1). At $37^{\circ} \mathrm{C}$, the PFP vapor pressure is about $132 \mathrm{kPa}$ [55], which is similar to the sum of the Laplace pressure after $(27 \mathrm{kPa})$ and the atmospheric pressure $(101 \mathrm{kPa})$. Thus, the gas bubble formed by ADV would be steady [22].

Another omnipresent and more likely hypothesis is that, the noncondensable gas (e.g., nitrogen, oxygen, etc.) dissolved in the surrounding liquid may diffuse to enter into the expanding PFC gas bubbles following ADV. Maybe the PFC gas contracts back to liquid during the high pressure phase of the acoustic cycle. But the noncondensable gas does not contract as a result leaving an extremely small gas bubble that will contribute to form a larger bubble on the next acoustic cycle. This process has been observed [56] and helps to manifest that the size of gas bubbles after cessation of ultrasound irradiation is much bigger than the size expected in the initial PFC droplets [57, 58].

\section{The Machanism of PFC Nanoparticles for Ultrasound Theranostics}

\section{Thermal Effects}

Generally, the heating produced in local tissues has been depended on all sorts of external stimuli, such as high intensity focused ultrasound (HIFU) $[59,60]$, radiofrequency (RF) [61, 62], microwave (MW) [62, 63], and laser [64], etc., which are devoted to thermal ablation of tumor tissues [65]. In addition, even a slight temperature, for instance, produced by ultrasound, may have effective biological consequences that dramatically enhance fluidization of cell membrane [66] and permeability of blood capillaries [67] through mechanical permeabilization of cell membranes.

The drugs loaded on temperature sensitive liposomes were rapidly released in the target region by thermal effects of ultrasound at physiologically temperatures as a result of heating to generate a transition of the phospholipid membrane from a gel to a fluid [6871]. In addition, the PFC nanoparticles encapsulated doxorubicin in combination with RF ablation have been receiving clinical trials $[69,72]$.

\section{Mechanical Action of Cavitation}

The cavitation of ultrasound can be significantly enhanced by the introduction of microbubbles converted by PFC nanoparticles. As a result, microbubbles have not only been used as contrast agents for ultrasound molecular imaging [73, 74], but also as carriers to enhance genes and drugs delivery [75-78].

On the one hand, the inertial cavitation of microbubbles grown and collapsed by mechanical force in the ultrasound field can produce shock waves and microjets that can create pores in cell membranes and blood vessels thereby accelerating the permeability of genes, drugs, and their carriers $[71,79,80]$. This phenomenon that inertial cavitation results in creation of cavities in cell membranes is called sonoporation [81, 82]. On the other hand, the process that microbubbles maintain smooth oscillation when the ultrasound energy is not enough to generate inertial cavitation is called stable cavitation, which can create commutative distention and invagination of blood vessel walls. As a result, the extraordinary permeability of blood vessel and damage of the endothelial lining have been enhanced [81, 83, 84] (Fig. 2).

Kheirolomoom et al. have reported that the development of ultrasound-reduced stable liposomes could prolong circulation time and accelerate tumor targeting [20, 86, 87]. Ultrasound-responsive cavitation has also been used to enhance permeabilization of cell membranes [88, 89]. Moreover, it has been demonstrated a probability to exceed the barrier produced by nuclear membrane in order to influence

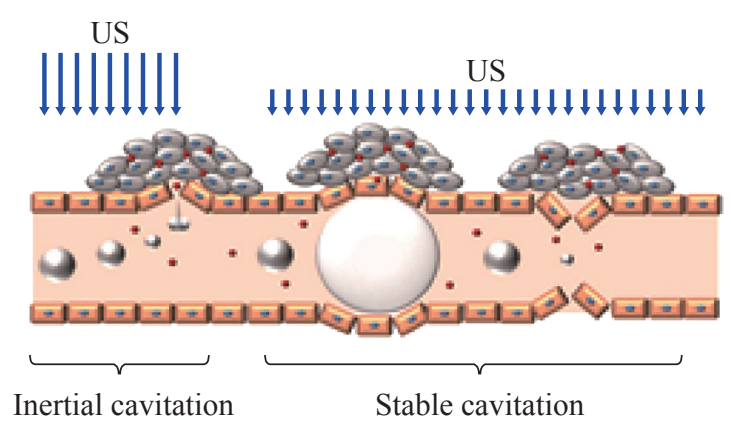

Fig. 2 The interactions between microbubbles and ultrasound. Extravasation of circulating drugs may produce by inertial cavitation events, causing to create pores in blood vessels and cell membranes. In the stable cavitation events, the smooth oscillation of microbubbles can create commutative distention and invagination of blood vessels, which also contribute to endothelial disruption and drug extravasation. The plot was adopted with permission from [85]. 
the intracellular drug distribution [90]. Drugloaded microbubbles would be attractive ultrasoundresponsive drug carriers. The approach that drugloaded microbubbles or phase-shift nanoparticles be beneficial for drug targeting to intravascular targets would be a dramatic ultrasound-responsive drug transportation [88, 89, 91-93].

\section{Mechanical Action of Non-cavitation}

The most regular arguments of non-thermal and non-cavitation mechanisms are correlated with ultrasound radiation force and acoustic streaming. The propagation of sound across a medium induces a force in the medium that can result in suspension of particles, called the radiation force, and also translation of fluid, called acoustic streaming [94, 95]. Both radiation force and acoustic streaming advance a method to concentrate microbubbles and nanoparticles near vessel walls, which may contribute to the delivery of targeted genes and drugs.

The application of radiation force can successfully bring the delivery agents into proximity of the cells and its fragments into cell membranes [96]. A similar research utilized for improving the cellular interaction of PFC nanoparticles elucidates that ultrasound incorporated both non-targeted and targeted PFOB nanoparticles showed security on the cell survival and no changes in cell monolayers [97]. Acoustic streaming and radiation force can also enhance extravasation of PFC nanoparticles, which are loaded genes and drugs, through blood capillary for molecular ultrasound imaging and therapy [94, 95, 98-100]. Additionally, another interesting application of ultrasound radiation force was found that it was used to regulate ligand exposure on the surface of targeted microbubbles [101]. The ligands hidden by droplet shells in the initial contrast agents had been transformed to the surface of droplet shells and exposed to the cell receptor under the action of ultrasound.

Eventually, the thermal and mechanical action of ultrasound on biological tissues increase concentration of contrast agents in targeted regions, enhance extravasation of drugs, genes and their carriers, and improve diffusion of microbubbles and nanoparticles passing varieties of biological barriers throughout tumor tissues. Thereby, the traditional therapeutic efficacy are dramatically increased as a result of the enhancement of intracellular uptake on nanoparticles, genes, and drugs [19, 29, 102-105].

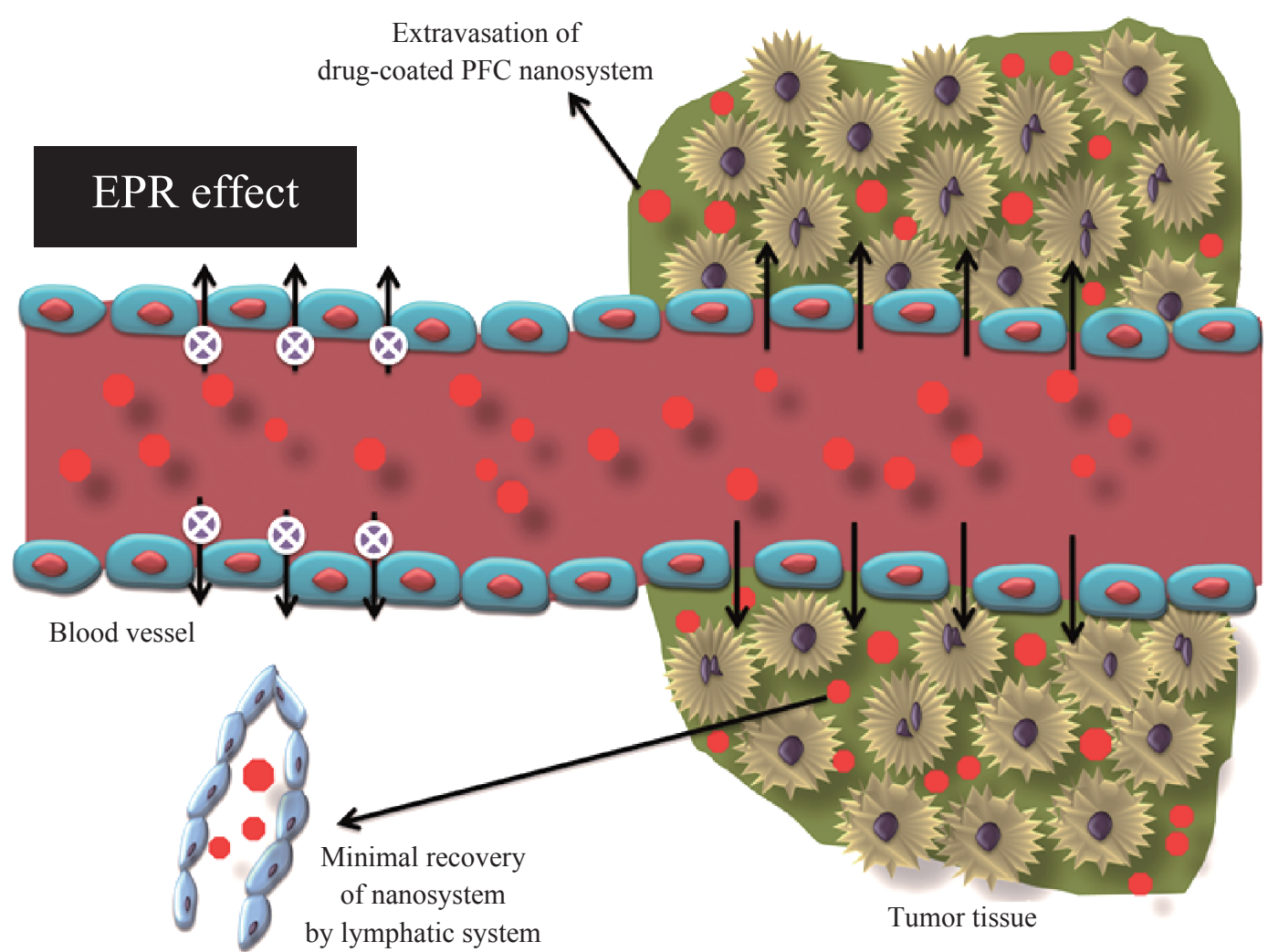

Fig. 3 Illustrative factors relating to the enhanced permeability and retention effect exhibited by tumor tissue. 


\section{The Mechanism of PFC Nanoparticles for Drug Delivery}

The characters of tumor tissue are irregular blood flow, poorly organized vascular architecture, poor vascularization, and reduced lymphatic drainage. The lack of a lymphatic system and leaky blood vessels increase the interstitial fluid pressure hampering delivery of drug carriers through blood vessel walls. However, the appropriate sized nanoparticles might accumulate in tumor tissue due to deficient tumor microvasculature system by the enhanced permeability retention (EPR) effect (illustrated in Fig. 3) [106]. Because of the characteristic pore cutoff size of interendothelial gaps of microvasculature system ranging from 380 to $780 \mathrm{~nm}$, it is feasible for drug-loaded nanoparticles to transport themselves across large inter-endothelial gaps in a vast variety of tumors [18, $106,107]$. On the contrary, the fact that blood vessels in normal tissues have tight inter-endothelial junctions whose characteristic cutoff size is just around $7.5 \mathrm{~nm}$ results in a prohibition of extravasation of drug-loaded nanoparticles.

Nevertheless, the spatial heterogeneity illustrated in the distribution of inter-endothelial gaps of tumor tissues leads to a regional distribution of delivered nanoparticles [23]. This may have negative connotation for the product of tumor nanotherapy. The capability of tumor targeting may be mainly accelerated by ligand/receptor mutual effect. For example, the PFC nanoparticles targets neovasculature and/or tumors through conjugating ligands to $\alpha_{v} \beta_{3}$ receptor, which is over expressed on the tumors, neoendothelial vasculature, and inflamed tissues [108$110]$.

Drug encapsulation in nanoparticles not only radically boosts the available aqueous solubility of extremely valid drugs whose application has been considerably bound with low solubility, but also could allow drug transport to expected targets thereby remarkably weakening side effects and hinder from drug degradation under the circulation of body fluids. Even the badly toxic and unstable pharmacon, such as the peptide melittin, was successful targeted to murine tumors due to be attached to PFC nanoparticles [111, 112]. As a result, the dramatically regressive tumors without noticeable systemic toxicity were observed.

\section{The Potential Application of PFC Nanoparticles in Medicine}

The panoply of molecular imaging applications for ultrasound can be elucidated with the extensive use of contrast agents. PFC nanoparticles exhibit a good safety profile with fever or flu-like symptoms in 24 hours after dosing $[24,26]$. There are no renal toxicity reported in human and animals [25]. With a nominal diameter of $250 \mathrm{~nm}$ around, PFC nanoparticles are not removed by the glomerular filtration but rather from the circulation by the reticuloendothelial system and then the PFC component ultimately is vaporized through respiration [27]. The blood clearance halflife of PFC nanoparticles between 3 and 42 hours due to the exact preparation method provides sufficient time for ultrasound imaging [113]. Here we review some indications for targeted and non-targeted PFC nanoparticles that are directly correlated with clinical medicine.

\section{Vascular Imaging}

Due to the enhancement of microbubbles on the back-scattered signal in ultrasound imaging, the first clinical application of phase-transition PFC nanoparticles emulsions as an ultrasound contrast agent seems to be in 1995 with a product called EchoGen [114], which was reported to be a suspension of albumin-encapsulated PFP nanoparticles with a diameter of $0.3 \mu \mathrm{m}$. And the sizes of transformational bubbles tended to be between 1 and $10 \mu \mathrm{m}$, with an average diameter of 6 to $8 \mu \mathrm{m}$ [16]. It could permit to enhance the contrast between the cortex and medulla of the kidney for the canine renal cortex imaging with an intravenous dose of 0.25 to $0.45 \mathrm{~mL} / \mathrm{kg}$ [114]. From then onward, extensively clinic applications of EchoGen had been validly investigated on ultrasound imaging with color Doppler analysis in human kidneys [115], left ventricular and myocardial opacification [16, 116-119], and prostates [120]. No side effects were observed and the safety evaluation of EchoGen (0.01 to $0.1 \mathrm{~mL} / \mathrm{kg}$ ) had been reported [121]. Besides that, another infusive imaging of EchoGen in basal cerebral arteries was covered in 1999 because that the nano-sized PFC emulsions retain a desirable high concentration for imaging where none agents had been done at that time [122].

Unfortunately, because of the competition of 
Definity, a microbubble contrast agent introduced in 1999 [123], EchoGen was not approved by the FDA in America, and apparent interest and funding vanished. However, the emulsions of PFC nanoparticle droplets still have been applied to other applications such as therapeutic delivery, vascular occlusion, and thrombosis.

\section{Cancer Imaging}

The conception of phase-transition PFC nanoparticles as drug carriers was initiated by Rapoport et al. In the system [124], the increase of temperature could lead PFC nanoparticles to vaporize into microbubbles that would generate larger microbubbles by coalescence of the vaporized droplets in the hypothermia of tumor region for ultrasound imaging and therapy. The phase-transition PFC nanoparticles as effective drug carriers were first reported in this study on account of the regression of tumor growth using PFP droplets to successfully deliver doxorubicin (DOX) into tumor tissues. And then, more valuable work has been explored by Rapoport [12, 19, 29, 103] et al. For instance, the PFC nanoemulsions encapsulated paclitaxel (PTX) can enhance the ultrasoundmediated tumor imaging on the ultrasonic therapy of pancreatic cancer and breast cancer in animal models due to the significant therapeutic characterizations based on tumor regression and suppression of metastasis [29].

Furthermore, the PFC nanoparticles can also be used for multiple modality imaging and enhancement of therapeutic efficiency of high intensity focused ultrasound (HIFU). In the most recent work, the application of enhancing ultrasound/magnetic resonance dual-modality imaging and accelerating the therapeutic efficiency of high intensity focused ultrasound (HIFU) has been observed in rabbit tumors after intravenous injection of gadolinium coated PFC nanoparticles [125]. Similar researches for multiple modality imaging and synergistic HIFU ablation of tumors are being investigated with PFC nanoparticles, such as the development of India ink incorporated phase-transition nanodroplets for photoacoustic/ ultrasound imaging and photoacoustic effect of tumor therapy [126], and a formulation of silica coated PFC nanoemulsion encapsulating camptothecin (CPT) for synergistic effect of chemotherapy and HIFU ablation in rabbit tumors [127].

\section{Thrombosis Imaging}

Because fibrin clots are scarce echogenic and thereby difficult to detect with ultrasound of clinical frequencies. Clinical ultrasound imaging of binding with PFC nanoparticles targeted to selected fibrin epitopes has been reported to detect the fibrin clots in vivo [17, 128, 129]. This could be useful for thrombotic diseases, such as acute kidney injury, sepsis, atherosclerosis, etc. Additionally, the detection of other constituents of thromboembolic disease has been demonstrated in ultrasound molecular imaging with targeted PFC nanoparticles. For example, Tissue Factor (TF), which is the proximate cause of thrombosis in plaque rupture and is also associated with microangiopathic thrombosis, is effectively detectable in injured vessel segments through the selective ultrasound contrast agents of PFC nanoparticles, which are targeted to TF using a specific conjugated monoclonal antibody fragment [130-133].

\section{Vascular Occlusion}

Embolotherapy is a method that is to occlude the vessel lacuna of the tissue with embolic materials such as microbubbles for treating tumors or other diseases of vascular malformations. Currently, PFC nanoparticles show promise for effectively targeted tissue occlusion $[38,134]$. In Zhang et al's study, substantial localized tissue occlusion was achieved in canine kidneys by gas bubbles in the renal arteries or segmental arteries, which were generated by phase-transition PFC droplets encapsulated in lipid shells or albumin in the blood [38]. Another study by the same group results that $125 \mu \mathrm{m}$ arterioles (average diameter) and the 4-7 $\mu \mathrm{m}$ capillary beds in Sprague-Dawley rats can be occluded with ultrasonically activated gas bubbles converted by albumin-encapsulated PFC nanoparticles (Samuel et al.) [135].

\section{Future perspective}

An unprecedented perspective in enhancement of clinic medicine is associated with developing ultrasound-responsive phase-transition PFC contrast agents on account of the inertial characters of low solubility and diffusivity. This novel technology delineates a potential value for the applications in ultrasound molecular imaging and therapy. The special 
property of PFC nanoparticles illustrates an excellent opportunity to diagnose, deliver therapy and predict therapeutic efficacy with response to ultrasound.

However, the PFC nanoparticles as contrast agents still have not been widely implemented at present. Much studies in vivo need to be proceeded for fetching phase-transition PFC nanoparticles in clinical medicine. Passive targeting action of PFC nanoparticles may be more challenging in larger animals and human on account of much smaller tumor-to-body volume ratio in large animals than in small animals. Selecting more appropriate receptors is also a critical mission for active targeting action. All of these issues remain to be performed in future researches.

In the above review, we have discussed a couple of mechanisms correlating with phase-transition PFC nanoparticles for ultrasound molecular imaging and therapy and their potential applications in ultrasound medicine. Although many of these still remain in the early stage of exploitation, they must have a remarkably potential utilization for diagnose and therapy in clinical applications.

\section{Acknowledgements}

Funding for this work was provided by the Program for Innovation Team Building at Institutions of Higher Education in Chongqing (No. KJTD201303) and by the National Natural Science Foundation of China (No. 81130025, No. 81371578, and No. 81401503).

\section{Financial \& Competing Interests Disclosure}

The authors report no conflicts of interest in this work.

\section{References}

[1] J.M. Yang, C. Favazza, R. Chen, et al., Simultaneous functional photoacoustic and ultrasonic endoscopy of internal organs in vivo. Nature medicine. 2012; 18: 1297 1302.

[2] J. Bohannon, Ultrasound uses in medicine heat up. Science (New York, NY). 2008; 321: 338-339.

[3] L.V. Wang, S. Hu, Photoacoustic tomography: in vivo imaging from organelles to organs. Science. 2012; 335: 1458-1462.

[4] E. Terreno, D.D. Castelli, A. Viale, et al., Challenges for molecular magnetic resonance imaging. Chemical reviews. 2010; 110: 3019-3042.
[5] W. Cai, X. Chen, Nanoplatforms for targeted molecular imaging in living subjects. Small. 2007; 3: 1840-1854.

[6] F. Yang, S. Hu, Y. Zhang, ,et al., A hydrogen peroxideresponsive $\mathrm{O}(2)$ nanogenerator for ultrasound and magnetic-resonance dual modality imaging. Advanced materials (Deerfield Beach, Fla). 2012; 24: 5205-5211.

[7] M.A. Nakatsuka, M.J. Hsu, S.C. Esener, et al., DNAcoated microbubbles with biochemically tunable ultrasound contrast activity. Advanced materials (Deerfield Beach, Fla). 2011; 23: 4908-4912.

[8] O.D. Kripfgans, J.B. Fowlkes, D.L. Miller, et al., Acoustic droplet vaporization for therapeutic and diagnostic applications. Ultrasound in medicine \& biology. 2000; 26: 1177-1189.

[9] E. Huynh, J.F. Lovell, B.L. Helfield,et al., Porphyrin shell microbubbles with intrinsic ultrasound and photoacoustic properties. Journal of the American Chemical Society. 2012; 134: 16464-16467.

[10] J. Zhang, R.J. Coulston, S.T. Jones, et al., One-step fabrication of supramolecular microcapsules from microfluidic droplets. Science (New York, NY). 2012; 335: 690-694.

[11] Y. Zhou, Z. Wang, Y. Chen, et al., Microbubbles from gasgenerating perfluorohexane nanoemulsions for targeted temperature-sensitive ultrasonography and synergistic HIFU ablation of tumors. Advanced materials. 2013; 25: 4123-4130.

[12] N. Rapoport, Phase-shift, stimuli-responsive perfluorocarbon nanodroplets for drug delivery to cancer. Wiley interdisciplinary reviews Nanomedicine and nanobiotechnology. 2012; 4: 492-510.

[13] Y. Chen, Q. Yin, X. Ji, et al., Manganese oxide-based multifunctionalized mesoporous silica nanoparticles for pH-responsive MRI, ultrasonography and circumvention of MDR in cancer cells. Biomaterials. 2012; 33: 71267137.

[14] H. Ke, J. Wang, Z. Dai, et al., Gold-nanoshelled microcapsules: a theranostic agent for ultrasound contrast imaging and photothermal therapy. Angewandte Chemie (International ed in English). 2011; 50: 3017-3021.

[15] M.S. Hughes, J.N. Marsh, H. Zhang, et al., Characterization of digital waveforms using thermodynamic analogs: detection of contrast-targeted tissue in vivo. Ultrasonics, Ferroelectrics and Frequency Control, IEEE Transactions on. 2006; 53: 1609-1616.

[16] P. Grayburn, Perflenapent emulsion (echogen ${ }^{\circledR}$ ): A new long-acting phase-shift agent for contrast echocardiography. Clinical cardiology. 1997; 20: 12-18.

[17] G.M. Lanza, S.A. Wickline, Targeted ultrasonic contrast agents for molecular imaging and therapy. Progress in cardiovascular diseases. 2001; 44: 13-31.

[18] R.B. Campbell, Tumor physiology and delivery of nanopharmaceuticals. Anti-Cancer Agents in Medicinal Chemistry (Formerly Current Medicinal Chemistry-AntiCancer Agents). 2006; 6: 503-512.

[19] N. Rapoport, A.M. Kennedy, J.E. Shea, et al., Ultrasonic nanotherapy of pancreatic cancer: lessons from ultrasound imaging. Molecular pharmaceutics. 2009; 7: 22-31.

[20] A. Kheirolomoom, L.M. Mahakian, C.Y. Lai, et al., Copper-Doxorubicin as a Nanoparticle Cargo Retains Efficacy with Minimal Toxicity. Molecular pharmaceutics. 2010; 7: 1948-1958.

[21] M.A. Hahn, A.K. Singh, P. Sharma, et al., Nanoparticles as contrast agents for in-vivo bioimaging: current status and future perspectives. Analytical and bioanalytical chemistry. 2011; 399: 3-27.

[22] M. Javadi, W.G. Pitt, C.M. Tracy, et al., Ultrasonic gene and drug delivery using eLiposomes. Journal of Controlled Release. 2013; 167: 92-100.

[23] M. Beck-Broichsitter, A.C. Dalla-Bona, T. Kissel, et al., 
Polymer Nanoparticle-Based Controlled Pulmonary Drug Delivery. Drug Delivery System: Springer; 2014, 133145.

[24] P.E. Keipert, S. Otto, S.F. Flaim, et al., Influence of perflubron emulsion particle size on blood half-life and febrile response in rats. Artificial cells, blood substitutes, and immobilization biotechnology. 1994; 22: 1169-1174.

[25] D.R. Spahn, Blood substitutes. Artificial oxygen carriers: perfluorocarbon emulsions. Critical care (London, England). 1999; 3: R93-97.

[26] R.J. Noveck, E.J. Shannon, P.T. Leese, et al., Randomized safety studies of intravenous perflubron emulsion. II. Effects on immune function in healthy volunteers. Anesthesia and analgesia. 2000; 91: 812-822.

[27] C.S. Cohn, M.M. Cushing, Oxygen therapeutics: perfluorocarbons and blood substitute safety. Critical care clinics. 2009;25:399-414, Table of Contents.

[28] N. Rapoport, D.A. Christensen, A.M. Kennedy, et al., Cavitation properties of block copolymer stabilized phaseshift nanoemulsions used as drug carriers. Ultrasound in medicine \& biology. 2010;36:419-429.

[29] N. Rapoport, K.H. Nam, R. Gupta, et al., Ultrasoundmediated tumor imaging and nanotherapy using drug loaded, block copolymer stabilized perfluorocarbon nanoemulsions. Journal of Controlled Release. 2011; 153: 4-15.

[30] G. Lanza, P. Winter, S. Caruthers, et al., Theragnostics for tumor and plaque angiogenesis with perfluorocarbon nanoemulsions. Angiogenesis. 2010; 13: 189-202.

[31] S. M. Janib, A.S. Moses, J.A. MacKay, Imaging and drug delivery using theranostic nanoparticles. Advanced drug delivery reviews. 2010; 62: 1052-1063.

[32] R.E. Apfel, Activatable infusable dispersions containing drops of a superheated liquid for methods of therapy and diagnosis. Google Patents; 1998.

[33] O.D. Kripfgans, J.B. Fowlkes, M. Woydt, et al., In vivo droplet vaporization for occlusion therapy and phase aberration correction. IEEE transactions on ultrasonics, ferroelectrics, and frequency control. 2002; 49: 726-738.

[34] O.D. Kripfgans, M.L. Fabiilli, P.L. Carson, et al., On the acoustic vaporization of micrometer-sized droplets. The Journal of the Acoustical Society of America. 2004; 116: 272-281.

[35] A.H. Lo, O.D. Kripfgans, P.L. Carson, et al., Acoustic droplet vaporization threshold: effects of pulse duration and contrast agent. IEEE transactions on ultrasonics, ferroelectrics, and frequency control. 2007; 54: 933-946.

[36] M.L. Fabiilli, K.J. Haworth, N.H. Fakhri, et al., The role of inertial cavitation in acoustic droplet vaporization. IEEE transactions on ultrasonics, ferroelectrics, and frequency control. 2009; 56: 1006-1017.

[37] M.L. Fabiilli, J.A. Lee, O.D. Kripfgans, et al., Delivery of water-soluble drugs using acoustically triggered perfluorocarbon double emulsions. Pharmaceutical research. 2010; 27: 2753-2765.

[38] M. Zhang, M.L. Fabiilli, K.J. Haworth, et al., Initial investigation of acoustic droplet vaporization for occlusion in canine kidney. Ultrasound Med Biol. 2010; 36: 1691-1703

[39] C.M. Carneal, O.D. Kripfgans, J. Krucker, et al., A tissue-mimicking ultrasound test object using droplet vaporization to create point targets. IEEE transactions on ultrasonics, ferroelectrics, and frequency control. 2011; 58: 2013-2025.

[40] M. Zhang, M.L. Fabiilli, K.J. Haworth, et al., Acoustic droplet vaporization for enhancement of thermal ablation by high intensity focused ultrasound. Academic radiology. 2011; 18: 1123-1132.

[41] O.D. Kripfgans, M. Zhang, M.L. Fabiilli, et al., Acceleration of ultrasound thermal therapy by patterned acoustic droplet vaporization. The Journal of the Acoustical Society of America. 2014; 135: 537-544.

[42] D.S. Li, O.D. Kripfgans, M.L. Fabiilli, et al., Formation of toroidal bubbles from acoustic droplet vaporization. Applied physics letters. 2014; 104: 063706.

[43] D.S. Li, O.D. Kripfgans, M.L. Fabiilli, et al., Initial nucleation site formation due to acoustic droplet vaporization. Applied physics letters. 2014; 104: 063703.

[44] P.C. Hiemenz, R. Rajagopalan, Principles of Colloid and Surface Chemistry, revised and expanded: CRC Press; 1997.

[45] P.S. Sheeran, T.O. Matsunaga, P.A. Dayton, Phasetransition thresholds and vaporization phenomena for ultrasound phase-change nanoemulsions assessed via high-speed optical microscopy. Physics in medicine and biology. 2013; 58: 4513.

[46] C.Y. Lin, W.G. Pitt, Acoustic droplet vaporization in biology and medicine. BioMed research international. 2013;2013.

[47] L.Y. Clasohm, I.U. Vakarelski, R.R. Dagastine, et al., Anomalous $\mathrm{pH}$ dependent stability behavior of surfactantfree nonpolar oil drops in aqueous electrolyte solutions. Langmuir. 2007; 23: 9335-9340.

[48] P.S. Sheeran, S. Luois, P.A. Dayton, et al., Formulation and acoustic studies of a new phase-shift agent for diagnostic and therapeutic ultrasound. Langmuir. 2011; 27: 10412-10420.

[49] P.S. Sheeran, S.H. Luois, L.B. Mullin, et al., Design of ultrasonically-activatable nanoparticles using low boiling point perfluorocarbons. Biomaterials. 2012; 33: 32623269.

[50] Z.Z. Wong, O.D. Kripfgans, A. Qamar, et al., Bubble evolution in acoustic droplet vaporization at physiological temperature via ultra-high speed imaging. Soft Matter. 2011; 7: 4009-4016.

[51] K.J. Haworth, J.B. Fowlkes, P.L. Carson, et al., Towards aberration correction of transcranial ultrasound using acoustic droplet vaporization. Ultrasound in medicine \& biology. 2008; 34: 435-445.

[52] S.T. Kang, C.K. Yeh, Intracellular Acoustic Droplet Vaporization in a Single Peritoneal Macrophage for Drug Delivery Applications. Langmuir. 2011; 27 :13183-13188.

[53] W.G. Pitt, R.N. Singh, K.X. Perez, et al., Phase transitions of perfluorocarbon nanoemulsion induced with ultrasound: a mathematical model. Ultrasonics sonochemistry. 2014; 21: 879-891

[54] A. Kabalnov, J. Weers, R. Arlauskas, et al., Phospholipids as emulsion stabilizers. 1. Interfacial tensions. Langmuir. 1995; 11: 2966-2974.

[55] R. Rowley, W. Wilding, J. Oscarson, et al., DIPPR Data Compilation of Pure Chemical Properties. http:// dipprbyuedu/. 2012.

[56] O. Shpak, L. Stricker, M. Versluis, et al., The role of gas in ultrasonically driven vapor bubble growth. Physics in medicine and biology. 2013; 58: 2523.

[57] N. Reznik, O. Shpak, E.C. Gelderblom, et al., The efficiency and stability of bubble formation by acoustic vaporization of submicron perfluorocarbon droplets. Ultrasonics. 2013; 53: 1368-1376.

[58] J. Lattin, D. Belnap, W. Pitt, Formation of eLiposomes as a drug delivery vehicle. Colloids and Surfaces B: Biointerfaces. 2012; 89: 93-100.

[59] J.W. Wijlemans, M. de Greef, G. Schubert, et al., A Clinically Feasible Treatment Protocol for Magnetic Resonance-Guided High-Intensity Focused Ultrasound Ablation in the Liver. Investigative radiology. 2015; 50: 24-31.

[60] S. Xu, Y. Zong, Y. Feng, et al., Dependence of pulsed focused ultrasound induced thrombolysis on duty cycle and cavitation bubble size distribution. Ultrasonics 
sonochemistry. 2015; 22: 160-166.

[61] B.J.J. Abdullah, C.H. Yeong, K.L. Goh, et al., Roboticassisted thermal ablation of liver tumours. European radiology. 2015; 25: 246-257.

[62] M.A. Chinnaratha, D. Sathananthan, P. Pateria, et al., High local recurrence of early-stage hepatocellular carcinoma after percutaneous thermal ablation in routine clinical practice. European Journal of Gastroenterology \& Hepatology. 2015.

[63] U. Leung, D. Kuk, M.D. Angelica, et al., Long-term outcomes following microwave ablation for liver malignancies. British Journal of Surgery. 2015; 102: 8591.

[64] K.C. Huang, W.T. Hsiao, C.H. Hwang, et al., The laser ablation model development of glass substrate cutting assisted with the thermal fracture and ultrasonic mechanisms. Optics and Lasers in Engineering. 2015; 67: 31-35.

[65] K.F. Chu, D.E. Dupuy, Thermal ablation of tumours: biological mechanisms and advances in therapy. Nature Reviews Cancer. 2014; 14: 199-208.

[66] T.M. Krupka, D. Dremann, A.A. Exner, Time and dose dependence of pluronic bioactivity in hyperthermiainduced tumor cell death. Experimental Biology and Medicine. 2009; 234: 95-104.

[67] M.R. Dreher, W. Liu, C.R. Michelich, et al., Tumor vascular permeability, accumulation, and penetration of macromolecular drug carriers. Journal of the National Cancer Institute. 2006; 98: 335-344.

[68] P.S. Yarmolenko, Y. Zhao, C. Landon, et al., Comparative effects of thermosensitive doxorubicin-containing liposomes and hyperthermia in human and murine tumours. International Journal of Hyperthermia. 2010; 26: 485-498

[69] Z. Vujaskovic, D.W. Kim, E. Jones, et al., A phase I/II study of neoadjuvant liposomal doxorubicin, paclitaxel, and hyperthermia in locally advanced breast cancer. International Journal of Hyperthermia. 2010; 26: $514-$ 521.

[70] A.H. Negussie, P.S. Yarmolenko, A. Partanen, et al., Formulation and characterisation of magnetic resonance imageable thermally sensitive liposomes for use with magnetic resonance-guided high intensity focused ultrasound. International Journal of Hyperthermia. 2011; 27: $140-155$.

[71] R. Deckers, C.T. Moonen, Ultrasound triggered, image guided, local drug delivery. Journal of Controlled Release. 2010; 148: 25-33.

[72] R.T. Poon, N. Borys, Lyso-thermosensitive liposomal doxorubicin: a novel approach to enhance efficacy of thermal ablation of liver cancer. Expert Opin Pharmacother. 2009; 10: 333-343.

[73] S. Song, H. Guo, Z. Jiang, et al., Self-Assembled Fe3O4/ Polymer Hybrid Microbubble with MRI/Ultrasound DualImaging Enhancement. Langmuir. 2014; 30: $10557-$ 10561.

[74] C. Tremblay-Darveau, R. Williams, L. Milot, et al., Combined perfusion and doppler imaging using planewave nonlinear detection and microbubble contrast agents. Ultrasonics, Ferroelectrics, and Frequency Control, IEEE Transactions on. 2014; 61: 1988-2000.

[75] Y. Yang, J. Wang, X. Li, et al., A near infrared fluorescent/ ultrasonic bimodal contrast agent for imaging guided pDNA delivery via ultrasound targeted microbubble destruction. RSC Advances. 2015; 5: 8404-8414.

[76] L.J.M, Juffermans, Ultrasound and microbubble-targeted delivery of drugs and genes: Cellular bioeffects and mechanisms. 2009.

[77] I. De Cock, E. Zagato, K. Braeckmans, et al., Ultrasound and microbubble mediated drug delivery: Acoustic pressure as determinant for uptake via membrane pores or endocytosis. Journal of Controlled Release. 2014.

[78] Z. Fan, R.E. Kumon, C.X. Deng, Mechanisms of microbubble-facilitated sonoporation for drug and gene delivery. Therapeutic delivery. 2014; 5: 467-486.

[79] O.F. Eker, B. Quesson, C. Rome, et al., Combination of cell delivery and thermoinducible transcription for in vivo spatiotemporal control of gene expression: a feasibility study. Radiology. 2011; 258: 496-504.

[80] G.A. Husseini, L. Kherbeck, W.G. Pitt, et al., Kinetics of ultrasonic drug delivery from targeted micelles. Journal of Nanoscience and Nanotechnology. 2015; 15: 20992104.

[81] I. Lentacker, I. De Cock, R. Deckers, et al., Understanding ultrasound induced sonoporation: Definitions and underlying mechanisms. Advanced drug delivery reviews. 2014; 72: 49-64.

[82] H. Yu, S. Chen, A model to calculate microstreamingshear stress generated by oscillating microbubbles on the cell membrane in sonoporation. Bio-medical materials and engineering. 2014; 24: 861-868.

[83] J.J. Rychak, A.L. Klibanov, Nucleic acid delivery with microbubbles and ultrasound. Advanced drug delivery reviews. 2014.

[84] E. Unger, T. Porter, J. Lindner, et al., Cardiovascular drug delivery with ultrasound and microbubbles. Advanced drug delivery reviews. 2014; 72: 110-126.

[85] S.R. Sirsi, M.A. Borden, State-of-the-art materials for ultrasound-triggered drug delivery. Advanced drug delivery reviews. 2014; 72: 3-14.

[86] E. Paoli, D. Kruse, J. Seo, et al., An optical and microPET assessment of thermally-sensitive liposome biodistribution in the Met-1 tumor model: Importance of formulation. Journal of Controlled Release. 2010; 143: 13-22.

[87] A. Kheirolomoom, D.E. Kruse, S. Qin, et al., Enhanced in vivo bioluminescence imaging using liposomal luciferin delivery system. Journal of Controlled Release. 2010; 141: $128-136$.

[88] A.L. Klibanov, T.I. Shevchenko, B.I. Raju, et al., Ultrasound-triggered release of materials entrapped in microbubble-liposome constructs: a tool for targeted drug delivery. Journal of Controlled Release. 2010; 148: 1317.

[89] S. Hernot, A.L. Klibanov, Microbubbles in ultrasoundtriggered drug and gene delivery. Advanced drug delivery reviews. 2008; 60: 1153-1166.

[90] P. Mohan, N. Rapoport, Doxorubicin as a molecular nanotheranostic agent: effect of doxorubicin encapsulation in micelles or nanoemulsions on the ultrasound-mediated intracellular delivery and nuclear trafficking. Molecular pharmaceutics. 2010; 7: 1959-1973.

[91] M. Böhmer, C. Chlon, B. Raju, et al., Focused ultrasound and microbubbles for enhanced extravasation. Journal of Controlled Release. 2010; 148: 18-24.

[92] F. Guenther, C. von zur Muhlen, E.A. Ferrante, et al., An ultrasound contrast agent targeted to P-selectin detects activated platelets at supra-arterial shear flow conditions. Investigative radiology. 2010; 45: 586.

[93] L.C. Phillips, A.L. Klibanov, D.K. Bowles, et al., Focused in vivo delivery of plasmid DNA to the porcine vascular wall via intravascular ultrasound destruction of microbubbles. Journal of vascular research. 2009; 47: 270-274.

[94] P. Dayton, A. Klibanov, G. Brandenburger, et al., Acoustic radiation force in vivo: a mechanism to assist targeting of microbubbles. Ultrasound in medicine \& biology. 1999; 25: 1195-1201.

[95] P.A. Dayton, S. Zhao, S.H. Bloch, et al., Application of ultrasound to selectively localize nanodroplets for targeted imaging and therapy. Molecular imaging. 2006; 5: 160. 
[96] M.J. Shortencarier, P.A. Dayton, S.H. Bloch, et al., A method for radiation-force localized drug delivery using gas-filled lipospheres. Ultrasonics, Ferroelectrics and Frequency Control, IEEE Transactions on. 2004; 51: 822831.

[97] N.R. Soman, J.N. Marsh, M.S. Hughes, et al., Acoustic activation of targeted liquid perfluorocarbon nanoparticles does not compromise endothelial integrity. NanoBioscience, IEEE Transactions on. 2006; 5: 69-75.

[98] C.K. Holland, D. D. McPherson, Echogenic lipsomes for targeted drug delivery. Biomedical Imaging: From Nano to Macro, 2009 ISBI'09 IEEE International Symposium on: IEEE; 2009; 755-758.

[99] S.M. Stieger, C.F. Caskey, R.H. Adamson, et al., Enhancement of Vascular Permeability with LowFrequency Contrast-enhanced Ultrasound in the Chorioallantoic Membrane Model. Radiology. 2007; 243: 112-121.

[100] K.W. Ferrara, Driving delivery vehicles with ultrasound. Advanced drug delivery reviews. 2008; 60: 1097-1102.

[101] A.F. Lum, M.A. Borden, P.A. Dayton, et al., Ultrasound radiation force enables targeted deposition of model drug carriers loaded on microbubbles. Journal of Controlled Release. 2006; 111: 128-134.

[102] N. McDannold, N. Vykhodtseva, K. Hynynen, Effects of acoustic parameters and ultrasound contrast agent dose on focused-ultrasound induced blood-brain barrier disruption. Ultrasound in medicine \& biology. 2008; 34: 930-937.

[103] N.Y. Rapoport, A.M. Kennedy, J.E. Shea, et al., Controlled and targeted tumor chemotherapy by ultrasound-activated nanoemulsions/microbubbles. Journal of Controlled Release. 2009; 138: 268-276.

[104] N.J. McDannold, N.I. Vykhodtseva, K. Hynynen, Microbubble Contrast Agent with Focused Ultrasound to Create Brain Lesions at Low Power Levels: MR Imaging and Histologic Study in Rabbits. Radiology. 2006; 241: 95-106.

[105] L.H. Treat, N. McDannold, N. Vykhodtseva, et al., Targeted delivery of doxorubicin to the rat brain at therapeutic levels using MRI - guided focused ultrasound. International journal of cancer. 2007; 121: 901-907.

[106] A.K. Iyer, G. Khaled, J. Fang, et al., Exploiting the enhanced permeability and retention effect for tumor targeting. Drug discovery today. 2006; 11: 812-818.

[107] S.K. Hobbs, W.L. Monsky, F. Yuan, et al., Regulation of transport pathways in tumor vessels: role of tumor type and microenvironment. Proceedings of the National Academy of Sciences. 1998; 95: 4607-4612.

[108] P.M. Winter, A.H. Schmieder, S.D. Caruthers, et al., Minute dosages of $\alpha_{v} \beta_{3}$-targeted fumagillin nanoparticles impair $\mathrm{Vx}-2$ tumor angiogenesis and development in rabbits. The FASEB Journal. 2008; 22: 2758-2767.

[109] P.M. Winter, S.D. Caruthers, H. Zhang, et al., Antiangiogenic synergism of integrin-targeted fumagillin nanoparticles and atorvastatin in atherosclerosis. JACC: Cardiovascular Imaging. 2008; 1: 624-634.

[110] H.F. Zhou, H.W. Chan, S.A. Wickline, et al., Alphavbeta3targeted nanotherapy suppresses inflammatory arthritis in mice. FASEB journal : official publication of the Federation of American Societies for Experimental Biology. 2009; 23: 2978-2985.

[111] N.R. Soman, S.L. Baldwin, G. Hu, et al., Molecularly targeted nanocarriers deliver the cytolytic peptide melittin specifically to tumor cells in mice, reducing tumor growth. The Journal of clinical investigation. 2009; 119: 2830-2842.

[112] N.R. Soman, G.M. Lanza, J.M. Heuser, et al., Synthesis and characterization of stable fluorocarbon nanostructures as drug delivery vehicles for cytolytic peptides. Nano letters. 2008; 8: 1131-1136.

[113] G. Hu, M. Lijowski, H. Zhang, et al., Imaging of Vx-2 rabbit tumors with alpha(nu)beta3-integrin-targeted 111In nanoparticles. International journal of cancer Journal international du cancer. 2007; 120: 1951-1957.

[114] C.M. Sehgal, P.H. Arger, C.R. Pugh, Sonographic enhancement of renal cortex by contrast media. Journal of ultrasound in medicine. 1995; 14: 741-748.

[115] J. Correas, S. Quay, EchoGen emulsion: a new ultrasound contrast agent based on phase shift colloids. Clinical radiology. 1996; 51: 11-14.

[116] J.D. Kasprzak, F.J. Ten Cate, New ultrasound contrast agents for left ventricular and myocardial opacification. Herz. 1998; 23: 474-482.

[117] P.A. Grayburn, J.L. Weiss, T.C. Hack, et al., Phase III multicenter trial comparing the efficacy of $2 \%$ dodecafluoropentane emulsion (EchoGen) and sonicated $5 \%$ human albumin (Albunex) as ultrasound contrast agents in patients with suboptimal echocardiograms. Journal of the American College of Cardiology. 1998; 32: 230-236.

[118] M.L. Main, P.A. Grayburn, Clinical applications of transpulmonary contrast echocardiography. American heart journal. 1999; 137: 144-153.

[119] W.G. Hundley, A.M. Kizilbash, I. Afridi, et al., Administration of an intravenous perfluorocarbon contrast agent improves echocardiographic determination of left ventricular volumes and ejection fraction: comparison with cine magnetic resonance imaging. Journal of the American College of Cardiology. 1998; 32: 1426-1432.

[120] H. Ragde, G. M. Kenny, G.P. Murphy, K. Landin, Transrectal ultrasound microbubble contrast angiography of the prostate. The Prostate. 1997; 32: 279-283.

[121] J.M. Correas, A.R. Meuter, E. Singlas, et al., Human pharmacokinetics of a perfluorocarbon ultrasound contrast agent evaluated with gas chromatography. Ultrasound in medicine \& biology. 2001; 27: 565-570.

[122] R. Totaro, M. Del Sette, C. Marini, Echocontrast agents in neurosonology. Functional neurology. 1998; 14: 235-239.

[123] K. Tiemann, S. Loheier, S. Kuntz, et al., Real-Time Contrast Echo Assessment of Myocardial Perfusion at Low Emission Power. Echocardiography. 1999; 16: 799809.

[124] N. Rapoport, Z. Gao, A. Kennedy, Multifunctional nanoparticles for combining ultrasonic tumor imaging and targeted chemotherapy. Journal of the National Cancer Institute. 2007; 99: 1095-1106.

[125] J.A. Kopechek, E. Park, C.S. Mei, et al., Accumulation of phase-shift nanoemulsions to enhance MR-guided ultrasound-mediated tumor ablation in vivo. Journal of healthcare engineering. 2013; 4: 109-126.

[126] J. Jian, C. Liu, Y. Gong, et al., India ink incorporated multifunctional phase-transition nanodroplets for photoacoustic/ultrasound dual-modality imaging and photoacoustic effect based tumor therapy. Theranostics. 2014; 4: 1026-1038.

[127] M. Ma, H. Xu, H. Chen, et al., A drug-perfluorocarbon nanoemulsion with an ultrathin silica coating for the synergistic effect of chemotherapy and ablation by highintensity focused ultrasound. Advanced materials. 2014; 26: 7378-7385.

[128] G.M. Lanza, C.H. Lorenz, S.E. Fischer, et al., Enhanced detection of thrombi with a novel fibrin-targeted magnetic resonance imaging agent. Academic radiology. 1998; 5 Suppl 1:S173-176; discussion S183-174.

[129] G.M. Lanza, K.D. Wallace, M.J. Scott, et al., A novel sitetargeted ultrasonic contrast agent with broad biomedical application. Circulation. 1996; 94: 3334-3340.

[130] G.M. Lanza, D.R. Abendschein, C.S. Hall, et al., In vivo molecular imaging of stretch-induced tissue factor in 
carotid arteries with ligand-targeted nanoparticles. Journal of the American Society of Echocardiography: official publication of the American Society of Echocardiography. 2000; 13: 608-614.

[131] G.M. Lanza, D.R. Abendschein, C.S. Hall, et al. Molecular imaging of stretch-induced tissue factor expression in carotid arteries with intravascular ultrasound. Invest Radiol. 2000; 35: 227-234.

[132] A. Morawski, P. Winter, S. Caruthers, et al., "Magnetic resonance immunocytochemistry": Characterization of "Tissue factor" expression by smooth muscle cells with targeted paramagnetic nanoparticles. Circulation: LIPPINCOTT WILLIAMS \& WILKINS 530 WALNUT ST, PHILADELPHIA, PA 19106-3621 USA; 2003,139139.

[133] G. Lanza, D. Abendschein, C. Hall, et al., In vivo molecular imaging of tissue factor in carotid arteries with a one-step ligand conjugated acoustic nanoparticle. Circulation: LIPPINCOTT WILLIAMS \& WILKINS 530
WALNUT ST, PHILADELPHIA, PA 19106-3621 USA; 1999, 72-72.

[134] N. Matsuura, R. Williams, I. Gorelikov, et al., Nanoparticle-loaded perfluorocarbon droplets for imaging and therapy. Ultrasonics Symposium (IUS), 2009 IEEE International: IEEE; 2009, 5-8.

[135] S. Samuel, A. Duprey, M.L. Fabiilli, et al., In vivo microscopy of targeted vessel occlusion employing acoustic droplet vaporization. Microcirculation. 2012; 19: 501-509.

Copyright $\odot 2015$ Jinshun Xu, Yang Cao, Chunyan Xu, Xuan Zhou, Jianxin Liu, Yuanzhi Yao, Pan Li, Li Zhou, Yufeng You, Lan Hao, Yang Sun, Weixiang Song, Yajing Zhao and Zhigang Wang. This is an open-access article distributed under the terms of the Creative Commons Attribution License, which permits unrestricted use, distribution, and reproduction in any medium, provided the original author and source are credited. 